\title{
The probability of patients being admitted from the emergency department is negatively correlated to in-hospital bed occupancy - a registry study
}

Mathias C Blom ${ }^{1 *}$, Fredrik Jonsson ${ }^{2}$, Mona Landin-Olsson ${ }^{1}$ and Kjell Ivarsson ${ }^{1}$

\begin{abstract}
Background: The association between emergency department (ED) overcrowding and poor patient outcomes is well described, with recent work suggesting that the phenomenon causes delays in time-sensitive interventions, such as resuscitation. Even though most researchers agree on the fact that admitted patients boarding in the ED is a major contributing factor to ED overcrowding, little work explicitly addresses whether in-hospital occupancy is associated to the probability of patients being admitted from the ED. The objective of the present study is to investigate whether such an association exists.
\end{abstract}

Methods: Retrospective analysis of data on all ED visits to Helsingborg General Hospital in southern Sweden between January 1, 2011, and December 31, 2012, was undertaken. The fraction of admitted patients was calculated separately for strata of in-hospital occupancy $<95 \%, 95-100 \%, 100-105 \%$, and $>105 \%$. Multivariate models were constructed in an attempt to take confounding factors, e.g., presenting complaints, age, referral status, triage priority, and sex into account. Subgroup analysis was performed for each specialty unit within the ED.

Results: Overall, 118,668 visits were included. The total admitted fraction was 30.9\%. For levels of in-hospital occupancy $<95 \%, 95-100 \%, 100-105 \%$, and $>105 \%$ the admitted fractions were $31.5 \%, 30.9 \%, 29.9 \%$, and $28.7 \%$, respectively. After taking confounding factors into account, the odds ratio for admission were $0.88(\mathrm{Cl} 0.84-0.93$, $P>0.001)$ for occupancy level $95-100 \%, 0.82(\mathrm{Cl} 0.78-0.87, P>0.001)$ for occupancy level $100-105 \%$, and 0.74 (Cl $0.67-0.81, P>0.001$ ) for occupancy level $>105 \%$, relative to the odds ratio for admission at occupancy level $<95 \%$. A similar pattern was observed upon subgroup analysis.

Conclusions: In-hospital occupancy was significantly associated with a decreased odds ratio for admission in the study population. One interpretation is that patients who would benefit from inpatient care instead received suboptimal care in outpatient settings at times of high in-hospital occupancy. A second interpretation is that physicians admit patients who could be managed safely in the outpatient setting, in times of good in-hospital bed availability. Physicians thereby expose patients to healthcare-associated infections and other hazards, in addition to consuming resources better needed by others.

Keywords: Bed occupancy; Emergency care; Patient admission

\footnotetext{
* Correspondence: mathias.blom@med.lu.se

${ }^{1} I \mathrm{KVL}$, Lund University, IKVL/Sektion I-II, Akutmedicin, Hs 32, EA-blocket, plan 2, Universitetssjukhuset, 22185 Lund, Sweden

Full list of author information is available at the end of the article
} 


\section{Background}

The association between emergency department (ED) overcrowding and poor patient outcomes is well described [1-7]. Recent work suggests that ED overcrowding compromises timeliness of resuscitative care, with potentially devastating effects to individual patients [8], and that ED overcrowding might have increased in magnitude over time [9]. Strategies to reduce overcrowding have been proposed [2,10-15] but their extent of implementation is variable $[4,14,16]$.

Boarding of admitted patients in the ED has been highlighted several times as the major cause of ED overcrowding [1,2,14,17-19]. Boarding is, in turn, caused by scarcity of inpatient beds $[1,2,14,16,17]$. Some consider ED overcrowding a symptom of the broader dysfunction in the healthcare system, rather than a problem residing solely in the ED [20-22]. Although not thoroughly investigated, full capacity protocols and other solutions aiming at distributing workload throughout the hospital instead of accumulating patients in the ED appear promising $[16,23]$. When asked, patients prefer boarding in inpatient hallways to ED hallways [24]. Other specific solutions to reduce boarding include synchronizing discharges from inpatient wards with admission peaks [25] and to eliminate bottlenecks delaying discharge, e.g., availability of surgery [26].

Admission is a comparably expensive intervention for ED patients, the use of which needs to be scrutinized in order to better understand cost-effectiveness in the evolving role of the EDs in Sweden and worldwide [21]. There is some evidence for physicians avoiding hospital admissions as an adaptive strategy in crowded conditions $[21,27]$, but few studies have explicitly addressed the correlation between in-hospital occupancy and the probability of admission. Beds are often scarce in Swedish hospitals, inciting the Swedish National Board of Health and Welfare to make the matter subject to national follow-up since 2012.

The objective of the present study is to test whether the probability of being admitted from the ED is correlated to the in-hospital occupancy at the time of patient presentation in the ED. The study is primarily hypothesisgenerating and constitutes part of an extensive project designed to elucidate the effects of high in-hospital occupancy on the treatment of acutely ill patients in the country council region of Skåne in southern Sweden.

\section{Methods}

\section{Study design}

This registry study included all visits to the ED of Helsingborg General Hospital registered in the ED information system Patientliggaren ${ }^{\oplus}$ between $1^{\text {st }}$ January 2011 and $31^{\text {st }}$ December 2012, not resulting in referral to another hospital. No further selection was made, in order not to introduce selection bias.

\section{Setting}

Helsingborg General Hospital is one of four emergency hospitals in the region of Skåne in southern Sweden. It is a 420-bed hospital with an ED serving a population of around 250,000. Due to tourism, the population expands to nearly 300,000 during the summer. The annual ED census is around 60,000 , with approximately $15 \%$ of patients arriving by ambulance. Patients are registered in the information system Patientliggaren ${ }^{\bullet}$ by a secretary upon arrival. Patients who arrive by ambulance or are referred by a physician gain access to the ED after registration. Nonreferred patients are given access to the ED after registration in accordance with pre-defined guidelines or are further evaluated by a nurse in primary triage. Such cases could be referred elsewhere (e.g., to primary care). About $10-15 \%$ of all visits enter the ED via primary triage. After entering the ED, secondary triage is performed by a nurse. The 4-level triage system "medical emergency triage and treatment system" was used during the study period. It addresses the medical urgency of a case by evaluating the main complaint and vital parameters. The triage priority number is registered in Patientliggaren ${ }^{\bullet}$ directly after secondary triage. Only physicians may down-prioritize patients.

Patients are directed to separate units for Surgery, Orthopaedics, Medicine, and Otolaryngology in a triageto-specialty model after secondary triage. A complementary unit staffed by emergency physicians capable of handling all complaints except for psychiatric, otolaryngologic, ophthalmologic, and paediatric (medicine) was also introduced in 2010 and operates from 8 am to $11 \mathrm{pm}$ daily. There are separate EDs for children with medical conditions ( $<18$ years of age) and for patients with obstetric/gynaecologic, psychiatric or ophthalmologic complaints. These are not included in the study. Patients with suspected hip-fractures and some geriatric patients with high acuity conditions are admitted directly and bypass the ED. Hand surgery, neurosurgery, and thoracic surgery is not available at the hospital. The availability of endovascular surgery and percutaneous coronary intervention is limited afterhours (between 17.00-08.00) and during weekends. Patients with these needs are referred to Skane University Hospital in Lund. Swedish national reimbursement systems are tied to a goal of $80 \%$ of visits with ED length of stay $\leq 4$ hours. ED length of stay at Helsingborg hospital is shorter than in academic EDs overseas $[4,28]$. Access to emergency care in Sweden is not dependent on private insurance status.

\section{Data sources}

Data on referral status was retrieved from the in-hospital information system PASiS. Data on all other variables was 
Table 1 Descriptive data

\begin{tabular}{|c|c|c|c|c|c|c|c|}
\hline & & All units & Medicine unit & Surgery unit & Orthopaedics unit & Emergency physician unit & Otolaryngology unit \\
\hline \multirow[t]{2}{*}{ Admission status } & Not admitted & $82,051(69.1 \%)$ & $18,493(52.8 \%)$ & $18,608(67.2 \%)$ & $23,484(85.1 \%)$ & $11,580(69.2 \%)$ & $9,886(85.2 \%)$ \\
\hline & Admitted & 36,617 (30.9\%) & $16,523(47.2 \%)$ & $9,102(32.8 \%)$ & $4,116(14.9 \%)$ & $5,160(30.8 \%)$ & $1,716(14.8 \%)$ \\
\hline \multirow[t]{4}{*}{ Age (yrs) } & $0-17$ & $16,381(13.8 \%)$ & 7,914 (22.6\%) & $4,846(17.5 \%)$ & $6,231(22.6 \%)$ & $1,747(10.4 \%)$ & $3,508(30.2 \%)$ \\
\hline & $18-39$ & 30,097 (25.4\%) & & $7,684(27.7 \%)$ & $7,442(27.0 \%)$ & $4,087(24.4 \%)$ & $3,019(26.0 \%)$ \\
\hline & $40-64$ & 33,468 (28.2\%) & $11,139(31.8 \%)$ & $7,328(26.4 \%)$ & $7,372(26.7 \%)$ & 4,855 (29.0\%) & 2,774 (23.9\%) \\
\hline & $>65$ & $38,722(32.6 \%)$ & $15963(45.6 \%)$ & $7,852(28.3 \%)$ & $6,555(23.8 \%)$ & $6,051(36.1 \%)$ & 2,301 (19.8\%) \\
\hline \multirow[t]{3}{*}{ Referral status } & Not referred & $91,168(76.8 \%)$ & 27,205 (77.7\%) & $22,187(80.1 \%)$ & $21,461(77.8 \%)$ & $12,698(75.9 \%)$ & $7,617(65.7 \%)$ \\
\hline & Referred & $18,667(15.7 \%)$ & $5,052(14.4 \%)$ & $3,379(12.2 \%)$ & 4,265 (15.5\%) & $3,156(18.9 \%)$ & $2,815(24.3 \%)$ \\
\hline & Missing & $8,833(7.4 \%)$ & $2,759(7.9 \%)$ & $2,144(7.7 \%)$ & $1,874(6.8 \%)$ & $886(5.3 \%)$ & $1,170(10.1 \%)$ \\
\hline \multirow[t]{5}{*}{ Triage priority } & 1 & $5,689(4.8 \%)$ & $3,298(9.4 \%)$ & $1,437(5.2 \%)$ & $277(1.0 \%)$ & $587(3.5 \%)$ & $90(0.8 \%)$ \\
\hline & 2 & $18,461(15.6 \%)$ & 9,136 (26.1\%) & 3,898 (14.1\%) & $2,181(7.9 \%)$ & $2,626(15.7 \%)$ & $620(5.3 \%)$ \\
\hline & 3 & $63,828(53.8 \%)$ & 17,423 (49.8\%) & $16,730(60.4 \%)$ & $15,522(56.2 \%)$ & $9,670(57.8 \%)$ & 4,483 (38.6\%) \\
\hline & 4 & 28,502 (24.0\%) & $4,774(13.6 \%)$ & $5,245(18.9 \%)$ & $8,703(31.5 \%)$ & $3,598(21.5 \%)$ & 6,182 (53.3\%) \\
\hline & Missing & $2,188(1.8 \%)$ & $385(1.1 \%)$ & $400(1.4 \%)$ & 917 (3.3\%) & $259(1.5 \%)$ & $227(2.0 \%)$ \\
\hline \multirow[t]{2}{*}{ Sex } & Female & $58,567(49.4 \%)$ & $18,156(51.9 \%)$ & $13,169(47.5 \%)$ & 13,232 (47.9\%) & $8,512(50.8 \%)$ & $5,498(47.4 \%)$ \\
\hline & Male & $60,101(50.6 \%)$ & $16,860(48.1 \%)$ & $14,541(52.5 \%)$ & 14,368 (52.1\%) & $8,228(49.2 \%)$ & 6,104 (52.6\%) \\
\hline \multirow[t]{4}{*}{ In-hospital occupancy } & $<95 \%$ & 53,405 (45.0\%) & $16,266(46.5 \%)$ & 13,129 (47.4\%) & 12,880 (46.7\%) & 6,391 (38.2\%) & 4,739 (40.8\%) \\
\hline & $95-100 \%$ & $34,258(28.9 \%)$ & $10,452(29.8 \%)$ & $8,070(29.1 \%)$ & 7,930 (28.7\%) & 4,558 (27.2\%) & 3,248 (28.0\%) \\
\hline & $100-105 \%$ & $23,920(20.2 \%)$ & 6,569 (18.8\%) & $5,167(18.6 \%)$ & $5,251(19.0 \%)$ & 4,296 (25.7\%) & $2,637(22.7 \%)$ \\
\hline & $>105 \%$ & 7,085 (6.0\%) & $1,729(4.9 \%)$ & $1,344(4.9 \%)$ & $1,539(5.6 \%)$ & $1,495(8.9 \%)$ & $978(8.4 \%)$ \\
\hline LWBS & Yes & 2,332 (2.0\%) & $692(2.0 \%)$ & $706(2.5 \%)$ & $571(2.1 \%)$ & $216(1.3 \%)$ & $147(1.3 \%)$ \\
\hline Passed primary triage & Yes & $18,616(15.7 \%)$ & 4,103 (11.7\%) & 4,198 (15.1\%) & $5,778(20.9 \%)$ & $2,678(16.0 \%)$ & 1,859 (16.0\%) \\
\hline Total visits & & 118,668 & 35,016 & 27,710 & 27,600 & 16,740 & 11,602 \\
\hline
\end{tabular}


retrieved from the ED information system Patientliggaren ${ }^{\circledR}$ Matching was performed by the hospital informatics unit using QlikView software. Data on in-hospital bed occupancy was retrieved from the informatics unit and was matched by the author $(\mathrm{MB})$ using $\mathrm{IBM}^{\odot} \mathrm{SPSS}^{\odot}$ Statistics 19. Data in Patientliggaren ${ }^{\circ}$ has been validated by the regional epidemiological unit "EpiCentrum" of the region of Skåne, as part of another project, in 2012-2013.

\section{Statistical analysis}

The admitted fraction was computed for strata of in-hospital occupancy of <95\%, 95-100\%, 100-105\%, and $>105 \%$. Subgroup analysis was performed for each specialty unit.

Binary logistic regression models were developed in an attempt to take confounding factors into account. Clinical judgement governed the decision of which predictors to screen for inclusion, but was inevitably tainted by data availability. Decisions were made a priori to analysis. Screened variables were the 10 most common presenting complaints, age-group, referral status, triage priority, presentation on a shift experiencing many visits (see definition below), presentation on night shift and during weekends, sex, leaving without being seen (LWBS), entering ED via primary triage, time to physician and in-hospital occupancy.

Age was grouped into intervals 0-18 years, $18-40$ years, 40-64 years, and $\geq 65$ years; youths in Sweden become of age at 18 and pension-age is 65 years. The youngest two age categories were merged in the analysis of the medicine unit, as children with medical conditions are assessed in a separate ED. In-hospital occupancy was categorized as <95\%, 95-100\%, 100-105\%, and >105\%. Presentation on a shift experiencing many visits was constructed as a dichotomous variable indicating presentation on one of the $25 \%$ of shifts subject to most visits (adjusted for shift type and unit). The night shift was set from 00.00 am to
08.00 am. Reference intervals for categorical predictors were in-hospital occupancy $<95 \%$, triage priority 3 , age $\geq 65$ years, presenting complaint other than the 10 most common, and not being referred to the ED. Since causes of missing data were not known, imputation was not considered an option and missing data was instead indicated by a separate category in each model [29].

Predictors were tested for crude association with the outcome before entering the preliminary primary effects model. Associations weaker than $P=0.25$, but of clinical importance, were still included [30]. Multicollinearity testing was performed using Spearman correlation [31]. Selection of interaction terms screened for inclusion in the final models was governed by clinical significance and terms were added to models one by one rather than stepwise [30]. Model fit was evaluated through Nagelkerke's $R^{2}$. The association between each predictor and the outcome were addressed by the -2LL and the Wald statistics. Models were screened for influential cases by addressing standardized residuals and Cook's distance.

Data was anonymized before entering SPSS. Ethical approval was granted by the ethics committee of Lund in February 6, dnr 2013/11.

\section{Results}

Between January 1, 2011 and December 31, 2012, 120,203 visits to the medicine, surgery, orthopaedics, emergency medicine, and otolaryngology units were registered in Patientliggaren $^{\circledR} ; 118,668$ visits did not result in referral to another hospital and were included in the study. Of these, 35,016 were directed to the medicine unit, 27,710 to the surgery unit, 27,600 to the orthopaedics unit, 16,740 to the emergency physician unit, and 11,602 to the otolaryngology unit. The admitted fraction was $30.9 \%$ overall, 47.2\% for the medicine unit, $32.8 \%$ for the surgery unit, $14.9 \%$ for the orthopaedics unit, $30.8 \%$ for the emergency

Table 2 Admitted fraction at different levels of in-hospital occupancy

\begin{tabular}{|c|c|c|c|c|c|c|}
\hline & & $<95 \%$ & $95-100 \%$ & $100-105 \%$ & $>105 \%$ & Total \\
\hline \multirow[t]{2}{*}{ All units } & Admitted & $16,845(31.5 \%)$ & $10,580(30.9 \%)$ & $7,159(29.9 \%)$ & $2,033(28.7 \%)$ & $36,617(30.9 \%)$ \\
\hline & Total & 53,405 & 34,258 & 23,920 & 7,085 & 118,668 \\
\hline \multirow[t]{2}{*}{ Medicine unit } & Admitted & $7,826(48.1 \%)$ & $4,831(46.2 \%)$ & $3,065(46.7 \%)$ & 801 (46.3\%) & $16,523(47.2 \%)$ \\
\hline & Total & 16,266 & 10,452 & 6,569 & 1,729 & 35,016 \\
\hline \multirow[t]{2}{*}{ Surgery unit } & Admitted & $4,358(33.2 \%)$ & $2,554(31.6 \%)$ & $1,718(33.2 \%)$ & $472(35.1 \%)$ & $9,102(32.8 \%)$ \\
\hline & Total & 13,129 & 8,070 & 5,167 & 1,344 & 27,710 \\
\hline \multirow[t]{2}{*}{ Orthopaedics unit } & Admitted & $1,959(15.2 \%)$ & $1,247(15.7 \%)$ & $694(13.2 \%)$ & $216(14.0 \%)$ & $4,116(14.9 \%)$ \\
\hline & Total & 12,880 & 7,930 & 5,251 & 1,539 & 27,600 \\
\hline \multirow[t]{2}{*}{ Emergency physician unit } & Admitted & $1,932(30.2 \%)$ & $1,424(31.2 \%)$ & $1,376(32.0 \%)$ & $428(28.6 \%)$ & $5,160(30.8 \%)$ \\
\hline & Total & 6,391 & 4,558 & 4,296 & 1,495 & 16,740 \\
\hline \multirow[t]{2}{*}{ Otolaryngology } & Admitted & 770 (16.2\%) & $524(16.1 \%)$ & 306 (11.6\%) & 116 (11.9\%) & 1,716 (14.8\%) \\
\hline & Total & 4,739 & 3,248 & 2,637 & 978 & 11,602 \\
\hline
\end{tabular}


physician unit, and $14.8 \%$ for the otolaryngology unit. Detailed descriptive data are reproduced in Table 1.

Unadjusted analysis showed that the admitted fraction was smaller in strata of increasing in-hospital occupancy. For levels of in-hospital occupancy <95\%, 95-100\%, 100$105 \%$, and $>105 \%$ the admitted fractions were $31.5 \%$, $30.9 \%, 29.9 \%$, and $28.7 \%$, respectively. The same pattern was observed in most of the subgroup analyses. Detailed results from the unadjusted analysis are reproduced in Table 2.

All predictors screened for inclusion in the multivariate models were included because of clinical significance, except from time to physician, which was omitted due to violation of the assumption of linearity in the logit [31].
Table 3 shows the association between in-hospital occupancy level and probability for admission, with confounding factors from presenting complaint, age group, referral status, triage priority, presentation on a shift experiencing many visits, presentation on night shift/weekend, sex, LWBS, and entering the ED via primary triage taken into account.

A clear association between increasing in-hospital occupancy and decreased odds ratio for admission was seen overall, with the odds ratios of admission being 0.88 (CI $0.84-0.93, P<0.001$ ) for occupancy level 95$100 \%, 0.82$ (CI $0.78-0.87, P<0.001$ ) for occupancy level $100-105 \%$, and 0.74 (CI $0.67-0.81, P<0.001$ ) for occupancy level $>105 \%$, relative to the odds ratio for admission

Table 3 Odds ratios for admission, with confounding factors taken into account

\begin{tabular}{|c|c|c|c|c|c|c|}
\hline & Occupancy level & B & SE & Wald & Sig. $(P)$ & OR (Cl) \\
\hline \multirow[t]{4}{*}{ All units } & $<95 \%$ (ref) & & & & & 1.00 \\
\hline & $95-100 \%$ & -0.12 & 0.025 & 24.26 & $<0.001$ & $0.88(0.84-0.93)$ \\
\hline & $100-105 \%$ & -0.20 & 0.029 & 47.17 & $<0.001$ & $0.82(0.78-0.87)$ \\
\hline & $>105 \%$ & -0.31 & 0.046 & 43.96 & $<0.001$ & $0.74(0.67-0.81)$ \\
\hline Nagelkerke R2 & 0.370 & & & & & \\
\hline \multirow[t]{4}{*}{ Medicine unit } & $<95 \%$ (ref) & & & & & 1.00 \\
\hline & $95-100 \%$ & -0.14 & 0.030 & 22.60 & $<0.001$ & $0.87(0.82-0.92)$ \\
\hline & $100-105 \%$ & -0.22 & 0.036 & 36.46 & $<0.001$ & $0.80(0.75-0.86)$ \\
\hline & $>105 \%$ & -0.33 & 0.060 & 29.86 & $<0.001$ & $0.72(0.64-0.81)$ \\
\hline Nagelkerke R2 & 0.367 & & & & & \\
\hline \multirow[t]{4}{*}{ Surgery unit } & $<95 \%$ (ref) & & & & & 1.00 \\
\hline & $95-100 \%$ & -0.16 & 0.035 & 20.35 & $<0.001$ & $0.85(0.80-0.91)$ \\
\hline & $100-105 \%$ & -0.17 & 0.042 & 17.11 & $<0.001$ & $0.84(0.78-0.91)$ \\
\hline & $>105 \%$ & -0.17 & 0.070 & 6.10 & 0.014 & $0.84(0.73-0.97)$ \\
\hline Nagelkerke R2 & 0.261 & & & & & \\
\hline \multirow[t]{4}{*}{ Orthopaedics unit } & $<95 \%$ (ref) & & & & & 1.00 \\
\hline & $95-100 \%$ & 0.004 & 0.049 & 0.006 & 0.940 & $1.00(0.91-1.11)$ \\
\hline & $100-105 \%$ & -0.26 & 0.061 & 18.62 & $<0.001$ & $0.77(0.68-0.87)$ \\
\hline & $>105 \%$ & -0.24 & 0.096 & 6.41 & 0.011 & $0.78(0.65-0.95)$ \\
\hline Nagelkerke R2 & 0.421 & & & & & \\
\hline \multirow[t]{4}{*}{ Emergency physician unit } & $<95 \%$ (ref) & & & & & 1.00 \\
\hline & $95-100 \%$ & -0.006 & 0.049 & 0.016 & 0.899 & $0.99(0.90-1.10)$ \\
\hline & $100-105 \%$ & -0.033 & 0.051 & 0.41 & 0.520 & $0.97(0.88-1.07)$ \\
\hline & $>105 \%$ & -0.25 & 0.075 & 10.60 & 0.001 & $0.78(0.68-0.91)$ \\
\hline Nagelkerke R2 & 0.357 & & & & & \\
\hline \multirow[t]{4}{*}{ Otolaryngology unit } & $<95 \%$ (ref) & & & & & 1.00 \\
\hline & $95-100 \%$ & 0.002 & 0.12 & 0.000 & 0.989 & $1.00(0.79-1.28)$ \\
\hline & $100-105 \%$ & -0.36 & 0.15 & 5.99 & 0.014 & $0.70(0.53-0.93)$ \\
\hline & $>105 \%$ & -0.40 & 0.22 & 3.44 & 0.063 & $0.67(0.44-1.02)$ \\
\hline Nagelkerke R2 & 0.287 & & & & & \\
\hline
\end{tabular}


at occupancy level $<95 \%$. A similar pattern was observed upon subgroup analysis. Table 3 shows the coefficients of overall fit.

\section{Discussion}

Both crude analysis and the adjusted analysis revealed a negative association between in-hospital occupancy and the odds ratio of patient admission. With few exceptions, the same pattern was observed upon subgroup analysis. The association between in-hospital occupancy level and decreased probability for admission established in the present study is supported by the findings of physicians aiming at reducing admissions when in-hospital beds are scarce [21,27].

Taking confounding from several factors into account, the multivariate models generated much information on factors associated with high probability of admission, which was omitted from this paper in order not to overshadow the main results. Most of the observed patterns were expected, i.e., increasing triage priority and age being associated with higher odds ratios of admission and different main complaints exhibiting different odds ratios for admission.

\section{Limitations}

Despite its place on the study design hierarchy, the authors believe that this retrospective descriptive study was good at approximating reality as it included a large population with a wide range of complaints of varying severity, being treated by typical personnel. No bias is introduced through selection, which might be the case in controlled studies [32]. Considering the prevailing savings requirements at Helsingborg General Hospital, a rapid and inexpensive approach was also considered most ethical. The external validity of the results has to be met with a sound measure of scepticism, as the study was performed in a single hospital. Future studies should address this subject by comparing results between the different hospitals in the region.

Goodness-of-fit statistics indicate a limited ability of the multivariate models to predict admissions, suggesting that variables not included play a role. Previous studies indicate that co-morbidity is an important factor [33] and the authors believe that vital parameters would be desirable to include in future models. It would also be interesting to include effects of queuing for radiology or laboratory resources. However, the size and completeness of the data material should eliminate some of the bias from these factors and the authors wish to point out that the study objective was to reveal any correlation between in-hospital occupancy and the probability for admission, not to develop a tool for predicting admissions. For this purpose, the chosen method is adequate [34]. Age was divided into fairly few intervals in order to minimize risk for incomplete information from the variables. Given the large number of cases, this approach was too cautious and possibly concealed interesting findings in the population $\geq 65$ years old.

\section{Conclusions}

High in-hospital occupancy is associated with decreased odds ratios for admission of patients presenting in the ED of Helsingborg General Hospital during the study period. One interpretation is that a fraction of patients who would benefit from inpatient care instead received suboptimal care in outpatient settings at times of high in-hospital occupancy. The authors propose that downstream effects of such a relationship would be measurable, e.g., by increased incidence of unplanned revisits to the ED.

A second interpretation is that physicians admit patients who could be managed safely in the outpatient setting, in times of good in-hospital bed availability. Physicians thereby expose patients to healthcare-associated infections and other hazards, in addition to consuming resources better needed by others. A study addressing the first of these hypotheses is already initiated.

\section{Competing interests}

The authors declare that they have no competing interests.

\section{Authors' contributions}

$\mathrm{MB}, \mathrm{KI}$ and $\mathrm{ML}$ all participated in developing the study protocol. MB carried out gathering and matching of data and also carried out the statistical analyses. MB carried out the writing of the draft. All authors carried out proofreading of repeated versions of the manuscript.

\section{Acknowledgements}

Thanks to the Laerdal Foundation, for the project grants which made the study possible. Thanks to Harvard Medical Faculty Physicians - Emergency Medicine Consulting, for inspiration and feedback on the methods section. Thanks to Lars Gustavsson, Helsingborg General Hospital Informatics Unit, for making data extracts from PASIS and matching data. Thanks to Ingemar Petersson, Epi-Centrum Skane, for his input regarding the statistics section and validation of the dataset.

\section{Author details}

${ }^{1}$ IKVL, Lund University, IKVL/Sektion I-II, Akutmedicin, Hs 32, EA-blocket, plan 2, Universitetssjukhuset, 22185 Lund, Sweden. ${ }^{2}$ Pre och intrahospital akutsjukvård, Helsingborgs lasarett, S Vallgatan 5, Helsingborg 251 87, Sweden.

Received: 3 September 2013 Accepted: 10 January 2014

Published: 5 February 2014

\section{References}

1. Morris ZS, Boyle A, Beniuk K, Robinson S: Emergency department crowding: towards an agenda for evidence-based intervention. Emerg Med J 2012, 29(6):460-466.

2. Wiler JL, Gentle C, Halfpenny JM, Heins A, Mehrotra A, Mikhail MG, Fite D: Optimizing emergency department front-end operations. Ann Emerg Med 2010, 55(2):142-160.

3. Guttmann A, Schull MJ, Vermeulen MJ, Stukel TA: Association between waiting times and short term mortality and hospital admission after departure from emergency department: population based cohort study from Ontario, Canada. BMJ 2011, 342:d2983.

4. Liu SW, Hamedani AG, Brown DF, Asplin B, Camargo CA Jr: Established and novel initiatives to reduce crowding in emergency departments. West J Emerg Med 2013, 14(2):85-89. 
5. Schull MJ, Vermeulen M, Slaughter G, Morrison L, Daly P: Emergency department crowding and thrombolysis delays in acute myocardial infarction. Ann Emerg Med 2004, 44(6):577-585.

6. Pines JM, Hollander JE, Localio AR, Metlay JP: The association between emergency department crowding and hospital performance on antibiotic timing for pneumonia and percutaneous intervention for myocardial infarction. Acad Emerg Med 2006, 13(8):873-878.

7. Richardson DB: Increase in patient mortality at 10 days associated with emergency department overcrowding. Med J Aust 2006, 184(5):213-216.

8. Hong KJ, Shin SD, Song KJ, Cha WC, Cho JS: Association between ED crowding and delay in resuscitation effort. Amer J Emerg Med 2013, 31(3):509-515.

9. Pitts SR, Pines JM, Handrigan MT, Kellermann AL: National trends in emergency department occupancy, 2001 to 2008: effect of inpatient admissions versus emergency department practice intensity. Ann Emerg Med 2012, 60(6):679-686.

10. McClelland MS, Lazar D, Sears V, Wilson M, Siegel B, Pines JM: The past, present, and future of urgent matters: lessons learned from a decade of emergency department flow improvement. Acad Emerg Med 2011, 18(12):1392-1399.

11. Ward MJ, Farley H, Khare RK, Kulstad E, Mutter RL, Shesser R, Stone-Griffith S: Achieving efficiency in crowded emergency departments: a research agenda. Acad Emerg Med 2011, 18(12):1303-1312.

12. Pham JC, Trueger NS, Hilton J, Khare RK, Smith JP, Bernstein SL: Interventions to improve patient-centered care during times of emergency department crowding. Acad Emerg Med 2011, 18(12):1289-1294.

13. Soremekun OA, Terwiesch C, Pines JM: Emergency medicine: an operations management view. Acad Emerg Med 2011, 18(12):1262-1268.

14. ACEP Boarding Task Force: Emergency department crowding: high-impact solutions, ACEP Task Force Report on Boarding. USA: American College of Emergency Physicians; 2008.

15. Howell E, Bessman E, Marshall R, Wright S: Hospitalist bed management effecting throughput from the emergency department to the intensive care unit. J Crit Care 2010, 25(2):184-189.

16. Gilligan P, Quin G: Full capacity protocol: an end to double standards in acute hospital care provision. Emerg Med J 2011, 28(7):547-549.

17. White BA, Biddinger PD, Chang Y, Grabowski B, Carignan S, Brown DF: Boarding inpatients in the emergency department increases discharged patient length of stay. J Emerg Med 2013, 44(1):230-235.

18. Hwang U, McCarthy ML, Aronsky D, Asplin B, Crane PW, Craven CK, Epstein SK Fee C, Handel DA, Pines JM, Rathlev NK, Schafermeyer RW, Zwemer FL Jr, Bernstein SL: Measures of crowding in the emergency department: a systematic review. Acad Emerg Med 2011, 18(5):527-538.

19. Bond K, Ospina MB, Blitz S, Afilalo M, Campbell SG, Bullard M, Innes G, Holroyd B, Curry G, Schull M, Rowe BH: Frequency, determinants and impact of overcrowding in emergency departments in Canada: a national survey. Healthcare Quarterly 2007, 10(4):32-40.

20. Pines JM: Emergency department crowding in California: a silent killer? Ann Emerg Med 2013, 61(6):612-614.

21. Kocher KE, Asplin BR: Emergency department crowding 2.0: coping with a dysfunctional system. Ann Emerg Med 2012, 60(6):687-691.

22. Richardson D, Kelly AM, Kerr D: Prevalence of access block in Australia 2004-2008. Emerg Medicine Australasia 2009, 21(6):472-478.

23. Villa-Roel C, Guo X, Holroyd BR, Innes G, Wong L, Ospina M, Schull M, Vandermeer B, Bullard MJ, Rowe BH: The role of full capacity protocols on mitigating overcrowding in EDs. Amer J Emerg Med 2012, 30(3):412-420.

24. Garson C, Hollander JE, Rhodes KV: Emergency department patient preferences for boarding locations when hospitals are at full capacity. Ann Emerg Med 2008, 51:9e12.

25. Emeny $R$, Vincent $C$ : Improved patient pathways can prevent overcrowding. Emerg Nurse 2013, 20(10):20-24.

26. Underwood R, Drachenberg J, Booth Thomas M, Cesta T: ED boarding creates patient safety issues, increases risk of mortality. Hosp Case Manag 2012, 21(3):29-31.

27. Nugus P, Holdgate A, Fry M, Forero R, McCarthy S, Braithwaite J: Work pressure and patient flow management in the emergency department: findings from an ethnographic study. Acad Emerg Med 2011, 18(10):1045-1052.

28. Anderson PD, Freitas R, Edlow JA, Tibbles C, Boyd K, Calder S: Blueprint for Change: Building a High Performance Emergency Care System in the Region Skane. Cambridge, MA: Harvard Medical Faculty Physicians; 2013.
29. von Elm E, Altman DG, Egger M, Pocock SJ, Gotzsche PC, Vandenbroucke JP: The Strengthening the Reporting of Observational Studies in Epidemiology (STROBE) statement: guidelines for reporting observational studies. Lancet 2007, 370(9596):1453-1457.

30. Hosmer DW, Lemeshow S: Applied Logistic Regression. 2nd edition. USA: Wiley; 2006.

31. Tabachnick B, Fidell LS: Using Multivariate Statistics. 5th edition. Boston: Pearson; 2006

32. Barrett TW, Schriger DL: Annals of Emergency Medicine Journal Club. Measures of emergency department crowding, odds ratios, and the dangers of making continuous data categorical. Answers to January 2008 journal club questions. Ann Emerg Med 2008, 51(6):782-789.

33. Sun Y, Heng BH, Tay SY, Seow E: Predicting hospital admissions at emergency department triage using routine administrative data. Acad Emerg Med 2011, 18(8):844-850.

34. Wiler $J$, Griffey RT, Olsen T: Review of modeling approaches for emergency department patient flow and crowding research. Acad Emerg Med 2011, 18(12):1371-1379.

doi:10.1186/1865-1380-7-8

Cite this article as: Blom et al.: The probability of patients being admitted from the emergency department is negatively correlated to in-hospital bed occupancy - a registry study. International Journal of Emergency Medicine 2014 7:8.

\section{Submit your manuscript to a SpringerOpen ${ }^{\odot}$ journal and benefit from:}

- Convenient online submission

- Rigorous peer review

- Immediate publication on acceptance

- Open access: articles freely available online

- High visibility within the field

- Retaining the copyright to your article

Submit your next manuscript at $>$ springeropen.com 